\title{
带对合的流形的协边类与其不动点集 的协边类间的关系
}

\author{
吴振德刘宗泽
}

（河北师范大学数学系,石家庄）

\section{一、引 文}

Stong 在文献 [1] 中曾指出不动点集的协边类不能决定带对合流形的任何东西. 本文讨 论了带对合的流形 $\left(M^{2 n-k}, T\right)(k=1,2,3,4)$, 其不动点集 $F^{n}$ 为常维数的情况. 主要结 果是

定理 对于任意的 $\alpha \in J_{2 n-k}^{n-k}, \beta \in I^{n-k}(k-1,2,3,4)$ 满足 $\chi(\alpha)=\chi(\beta)$, 则存在 $\left(M^{2 n-k}, T\right)$, 其不动点集为 $F^{n}$, 使得 $\left[M^{2 n-k}\right]=\alpha,\left[F^{n}\right]=\beta$.

下面把文中的符号说明如下: $\mathscr{R}_{n}$ 表示 $n$ 维不定向的协边群, [ $\left.M^{2 n-k}\right]$ 表示 $M$ 所属的协 边类; $J_{2 n-k}^{n-k}=\left\{\left[M^{2 n-k}\right] \mid\left(M^{2 n-k}, T\right)\right.$ 的不动点集为常 维数 $\left.n\right\} ; I^{n-k}=\left\{\left[F^{n}\right] \mid F^{n}\right.$ 是某个 $\left(M^{2 n-k}, T\right)$ 的不动点集); $\chi_{n}=\left\{\left[M^{n}|| \chi\left(M^{n}\right)=0\right\} ; \chi(M)\right.$ 表示 $M$ 的模 2 的 Euler 数. 其 它符号同文献 $[1,2]$. 文中所采用的系数域为 $Z_{2} . R P(n)$ 代表 $n$ 维实射影空间。

\section{二、定理的证明}

引理 1 (文献[ 2 ]引理 2.1)

$$
\bigcup_{＝ 0}^{m} \nu^{m-i} \rightarrow F^{i} \text { 是 } m-i \text { 维向量丛的不交并. } \propto \text { 为 } \mathscr{R}_{m} \text { 中 }
$$
的元素. 存在带对合的流形 $\left(V^{m}, T\right)$ 使得 $\left[V^{m}\right]-\alpha$ 且 $\bigcup_{=0}^{m} \nu^{m-i} \rightarrow F^{\prime}$ 为其不动点的法丛的 充分必要条件是 $J^{\prime}\left(\sum_{j=0}^{m}\left[\nu^{m-i} \rightarrow F^{i}\right]\right)=\beta Q^{m}+$ 高次项.

本引理在文献 [2] 中已证明, $J$ 的意义见文献 [2].

引理 2 若 $\left[F^{*}\right]$ 为 $\mathscr{R}_{n}$ 中不可分解元且 $w_{i}\left(F^{*}\right)-0, i>\alpha(n)+1$, 则存在 $\left(M^{2 n-k}\right.$, $T), n-k \geqslant \alpha(n)+1$, 其不动点集为 $F^{\prime}$, 使得 $\left[F^{\prime}\right]-\left[F^{*}\right],\left[M^{2 n-k}\right]-0$.

证 (参见文献 $\left[21\right.$ 引理 3、4 的证明) 由于 ( $F^{n} \times F^{n}$, twist) 的不动点集的法丛为 $\tau F^{n} \rightarrow F^{n}$, 又根据 $w_{i}\left(F^{n}\right)=0, i>\alpha(n)+1$, 于是有 $\left(\xi^{n-k} \oplus k R \rightarrow F^{\prime}\right) \sim\left(\tau F^{n} \rightarrow F^{*}\right)$ 。 其中 $n-k \geqslant a(n)+1$. 又因

$$
\begin{aligned}
J\left(\xi^{n-k} \rightarrow F^{\prime}\right) & =J^{\prime}\left(\xi^{n-k} \oplus k R \rightarrow F^{\prime}\right) \\
& =J^{\prime}\left(\tau F^{n} \rightarrow F^{n}\right) \\
& =\left[F^{n} \times F^{n}\right] Q^{2 n}+\text { 高次项。 }
\end{aligned}
$$

本文 1987 年 6 月 1 日收到。 
根据引理 1 , 有 $\left(M^{2 n-k}, T\right)$, 不动点集为 $F^{\prime}$, 且 $\left[M^{2 n-k}\right]=0$. 由于 $\left(\xi^{n-k} \oplus k R \rightarrow F^{\prime}\right) \sim$ $\left(\tau F^{n} \rightarrow F^{*}\right)$ ，自然有 $F^{\prime} \sim F^{n}$.

引理 3 若 $\left(M^{2 l-k}, T\right)$ 的不动点集的协边类为 $\alpha_{l},\left[M^{2 l-k}\right]=0, \beta_{m}$ 为 $\mathscr{R}_{m}$ 中任意 元, 则存在 $\left(N^{2(m+l)-k}, T^{\prime}\right)$, 其不动点集的协边类为 $\beta_{m} \cdot \alpha_{l}$, 且 $\left[N^{2(m+l)-k}\right]=0$.

证 取 $\left(N^{2(m+l)-k}, T^{\prime}\right)=\left(B^{m} \times B^{m} \times M^{2 l-k}\right.$, twist $\left.\times T\right)$ 即可. 其中 $\left[B^{m}\right]=\beta_{m}$.

引理 $4 \quad R P\left(n_{1}, n_{2}, \cdots n_{2 k}\right)$ 有对合 $T^{*}$, 使其不动点集为 $R P\left(\Lambda_{1} \oplus \cdots \oplus \ell_{k} \rightarrow R P\left(n_{1}\right) \times\right.$ $\left.\cdots \times R P\left(n_{2 k}\right)\right) \cup R P\left(\lambda_{k+1} \oplus \cdots \oplus \Lambda_{2 k} \rightarrow R P\left(n_{1}\right) \times \cdots \times R P\left(n_{2 k}\right)\right)$

证明参见文献 [3], p. 160 .

命题 1 对任意的 $\alpha \in J_{2 n-1}^{n-1}, \beta \in I_{n}^{n-1}=\chi_{n}$, 存在 $\left[M^{2 n-1}, T\right]$, 其不动点集为 $F^{n}$, 满 足 $\left[M^{2 n-1}\right]=\alpha,\left[F^{n}\right]=\beta$.

证 由文献 [2] 引理 3.6 可知, 当 $n>2$, 且 $\neq 2^{r}-1$ 时 $\mathscr{R}_{n}$ 中存在不可分解元 $x_{n}$, 满足 $w_{i}\left(x_{n}\right)=0, i>\alpha(n)+1$, 现称此类元素为规范不可分解元.

取 $x_{n}\left(n>2\right.$ 且 $\left.\neq 2^{\prime}-1\right)$ 为规范不可分解元, 取 $x_{2}=R P(2)$, 它们构成了 $\mathscr{R}_{*}$ 的一 组环基. 由引理 2 可知对任 $n \geqslant 4$, 有 $n-1>\alpha(n)+1$, 于是有 $\left(M^{2 n-1}, T\right)$, 其不动点 集为 $F^{n}$, 使得 $\left[F^{n}\right]-x_{n},\left[M^{2 n-1}\right]=0$.

又对任意的 $\gamma_{m} x_{l}$, 其中 $l \geqslant 4, m+l=n$, 取 $N^{m}, F^{l}$ 分别为 $x_{m}, x_{l}$ 的代表. 取 $\left(V^{2 l-1}\right.$, $T$ ) 使其不动点集为 $F^{l}$, 且 $\left[V^{2 l-1}\right]=0$ (由上面讨论可知这是可以做到的), 则 ( $N^{m} \times N^{m} \times$ $V^{2 l-1}$, twist $\left.\times T\right)$ 之不动点集为 $N^{m} \times F^{l}$ ，且 $\left[N^{m} \times N^{m} \times V^{2 l-1}\right]=0$. 因为 $\chi_{n}$ 中的元 皆为含 $x_{n}(n \geqslant 4)$ 的一些乘积的和, 从而可知对任意的 $\beta_{n} \in I_{n}^{n-1}=\chi_{n}$, 皆存在 $\left(M^{2 n-1}, T\right)$, 其不动点集为 $F^{n}$, 使得 $\left[F^{n}\right]=\beta_{n},\left[M^{2 n-1}\right]=0$.

对任 $\alpha \in J_{2 n-1}^{n-1}, \beta \in I_{n}^{n-1}$, 存在 $\left(M_{1}^{2 n-1}, T_{1}\right)$, 满足 $\left[M_{1}^{2 n-1}\right]=\alpha$, 设其不动点集为 $F_{1}^{n}$; 可以取 $\left(M_{2}^{2 n-1}, T_{2}\right)$ 使其不动点集为 $F_{2}^{n}$, 满足 $\left[F_{2}^{n}\right]=\beta$ ，且 $\left[M_{2}^{2 n-1}\right]=0$; 取 $\left(M_{3}^{2 n-1}, T_{3}\right)$ 使其不动点集为 $F_{3}^{n}$, 满足 $\left[F_{3}^{n}\right]=\left[F_{1}^{n}\right]$ 且 $\left[M_{3}^{2 n-1}\right]=0$. 作 $(M, T)=\left(M_{1} \cup M_{2} \cup M_{3}\right.$, $\left.T_{1} \cup T_{2} \cup T_{3}\right)$ ，则其不动点集的协边类为 $\beta$ ，且 $[M]=\alpha$.

命题 2 对任 $\alpha \in J_{2 n-2}^{n-2}, \beta \in I_{n}^{n-2}, \chi(\alpha)=\chi(\beta)$, 则存在 $\left(M^{2 n-2}, T\right)$, 其不动点集为 $F^{n}$, 使得 $\left[M^{2 n-2}\right]=\alpha,\left[F^{n}\right]=\beta$.

证 取 $x_{n}\left(n \geqslant 4\right.$ 且 $\left.\neq 2^{\prime}-1\right)$ 为规范不可分解元. $x_{2}=R P(2)$. 由于 $n \geqslant 4$, 有 $n-2 \geqslant \alpha(n)+1$. 由引理 2 可知, 对任 $x_{n}(n \geqslant 4)$, 有 $\left(M^{2 n-2}, T\right)$ 其不动点集为 $F^{n}$, 满 足 $\left[F^{n}\right]=x_{n},\left[M^{2 n-2}\right]=0$. 又 $\chi_{n}$ 中的元必为含 $x_{n}(n \geqslant 4)$ 的一些乘积的和, 依照命题 1 的方法,可以得到当 $\chi(\alpha)=\chi(\beta)=0$ 时,命题 2 成立.

当 $\chi(\alpha)=\chi(\beta)=1$ 时, 取 $\alpha^{\prime}=\alpha+\left[R P^{n-1}(2)\right], \beta^{\prime}=\beta+\left[R P^{n}(2)\right]$ (因为 $\chi(\beta)=$ 1 , 故 $n$ 为偶数 $)$, 依前可得 $\left(M_{1}^{2 n-2}, T_{1}\right)$ ，其不动点集为 $F_{\mathrm{l}}^{n}$ 满足 $\left[M_{1}^{2 n-2}\right]=\alpha^{\prime},\left[F_{1}^{*}\right]=B^{\prime}$. 作 $\left(M_{2}^{2 n-2}, T_{2}\right)=\left(R P^{\frac{n}{2}-1}(2) \times R P^{\frac{n}{2}-1}(2) \times R P(2)\right.$, twist $\left.\times 1\right)$, 其不动点集为 $R P_{2}^{n}(2)$. 于是作 $\left(M_{1} \cup M_{2}, T_{1} \cup T_{2}\right)$ ，即可证明命题之结论.

命题 3 对任 $\alpha \in J_{2 n-3}^{n-3} \beta \in I_{n}^{n-3}=\chi_{n}$, 则存在 $\left(M^{2 n-3}, T\right)$, 其不动点集为 $F^{n}$, 使得 $\left[M^{2 n-3}\right]=\alpha,\left[F^{n}\right]=\beta$.

证 作规范不可分解元 $x_{n}\left(n \geqslant 5\right.$ 且 $\left.\neq 2^{\prime}-1\right)$, 令 $x_{2}-R P(2)$. 当 $n \geqslant 6$ 时有 $n-$ $3 \geqslant \alpha(n)+1$, 依引理 2 , 有 $\left[M^{2 n-3}, T\right)$, 且不动点集为 $F^{n}$, 使得 $\left[M^{2 n-3}\right]=0,\left[F^{n}\right]=x_{n}$.

由于 $I_{5}^{k}=\mathscr{R}_{5}, x_{5} \in I_{5}^{2}$, 故有 $\left(M^{7}, T\right)$ 其不动点集的协边类为 $x_{5}$. 若 [ $\left.M^{7}\right]=0$, 则 
$M^{7}$ 即为所求. 若 $\left[M^{7}\right] \neq 0$, 作 $\left(R P(2,0,0,0) \times R P(2), T^{*} \times 1\right)$ 则其不动点集 为 $(R P(2,0) \cup R P(2) \times R P(1)) \times R P(2)$, 其协边类为 $[R P(2,0) \times R P(2)]-0$. 又因 $[R P(2,0,0,0) \times R P(2)] \neq 0$, 且 $\mathscr{R}$, 中仅有一个非零元, 故 $[R P(2,0,0,0) \times R P(2)$ $\left.U M^{7}\right]=0$. 而 $\left(R P(2,0,0,0) \times R P(2) \cup M^{7}, T^{*} \times 1 \cup T\right)$ 之不动点集的协边类为 $x_{5}$.

取 $x_{4}=R P(1,1,0)$, 此为 $\mathscr{R}_{4}$ 中的不可分解元, 又 $\left(R P(1,1,0,0,0,0), T^{*}\right)$ 之不 动点集的协边类为 $[R P(1,1,0)]$ 且 $[R P(1,1,0,0,0,0)]=0$, 作 $(M, T)=(R P(1$, $\left.1,0,0,0,0) \times R P(2), T^{*} \times 1\right)$, 有 $[M]=0$, 其不动点集的协边类为 $x_{2} \cdot x_{4}$, 而 $I_{n}^{:-3}$ 中 的元素皆由 $x_{2} \cdot x_{4}$ 及 $x_{l}(l \geqslant 5)$ 生成,依照前面的方法,可以证明命题 3.

命题 4 对任 $\alpha \in J_{2 n-4}^{n-4}, \beta \in I_{n}^{n-4}, \chi(\alpha)=\chi(\beta)$, 则存在 $\left(M^{2 n-4}, T\right)$, 其不动点集为 $F^{n}$, 使得 $\left[M^{2 n-4}\right]=\alpha,\left[F^{n}\right]=\beta$.

证 作 $\mathscr{R}_{*}$ 之规范不可分解元 $x_{n}\left(n=4,5\right.$, 及 $n \geqslant 8$ 且 $\left.\neq 2^{r}-1\right)$. 取 $x_{2}-R P(2)$, $x_{6}=[R P(6)]+\left[R P^{3}(2)\right]$ 、于是当 $n \geqslant 8$ 时有 $n-4 \geqslant \alpha(n)+1$. 依引理 2, 有 $\left(M^{2 n-4}, T\right)$ 其不动点集为 $F^{*}$, 使得 $[M]=0,\left[F^{n}\right]=x_{n}$.

依引理 3 及命题 2 , 对 $x_{2} \cdot x_{4}, x_{2} \cdot x_{3}, x_{4}^{2}, x_{4} \cdot x_{3}, x_{5}^{2}$ 分别有相应的 $(M, T)$, 其不动点集 的协边类分别为 $x_{2} \cdot x_{4}, x_{2} \cdot x_{3}, x_{4}^{2}, x_{4} \cdot x_{5}, x_{3}^{2}$ 且 $[M]=0$.

现考虑 $x_{0}$, 由文献 [2] 341 页引理 2.3 可知, 对于 $R P(6,0,0) \cup R P(3,3,0)$ 存在对合, 使其不动点集的协边类为 $[R P(6)]$. 又 $[R P(6,0,0) \cup R P(3,3,0)]=\left[R P^{2}(4)\right], R P^{2}(2) \times$ $R P(2) \times R P(2)$, twist $\times 1 \times 1)$ 之不动点集为 $R P^{3}(2)$. 故 $\left[R P^{2}(4) \cup R P^{4}(2)\right]$ 中有代表 $M$, 可取适当的对合使其不动点集的协边类为 $\left[R P(6) \cup R P^{3}(2)\right]$, 又 $[R P(4,1,0,0)]=$ $\left[R P^{3}(4) \cup R P^{4}(2)\right]$, 而 $\left(R P(4,1,0,0), T^{*}\right.$ 之不动点集的协边类为零. 从而在 $[R P(4$, $\left.1,0,0) \cup R P^{2}(4) \cup R P^{4}(2)\right]$ 中有代表 $N$, 对 $N$ 可取适当的对合其不动点集的协边类为 $x_{6}$, 而 $\left[R P(4,1,0,0) \cup R P^{3}(4) \cup R P^{4}(2)\right]=0$. 依照前面的方法, 可以证明命题 4 成立.

综合上述各命题,即得本文之定理。

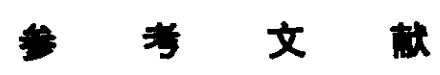

[1] Kosniowski, C., Stong, R. E., Topology, 17(1978), 309-330.

[2] Capobianco, F.L., Paci. J. of Math., 75(1978), 2: 339-345.

[3] , Proc. of Amer. Math. Soc., 61(1976), 157-162. 